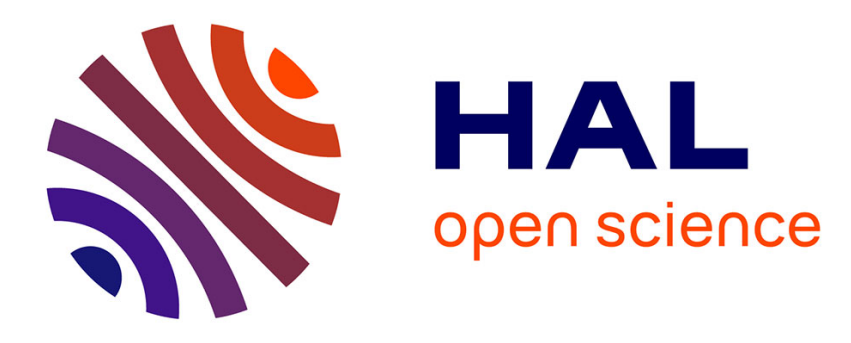

\title{
Aulu-Gelle, Probus et le problème de la traduction des textes poétiques \\ Alessandro Garcea
}

\section{To cite this version:}

Alessandro Garcea. Aulu-Gelle, Probus et le problème de la traduction des textes poétiques. Bernard Bortolussi; Madeleine Keller; Sophie Minon; Lyliane Sznajder. Traduire, transposer, transmettre dans l'Antiquité gréco-romaine, Picard, pp.17-26, 2009. halshs-00402857

\section{HAL Id: halshs-00402857 https://shs.hal.science/halshs-00402857}

Submitted on 13 Apr 2019

HAL is a multi-disciplinary open access archive for the deposit and dissemination of scientific research documents, whether they are published or not. The documents may come from teaching and research institutions in France or abroad, or from public or private research centers.
L'archive ouverte pluridisciplinaire HAL, est destinée au dépôt et à la diffusion de documents scientifiques de niveau recherche, publiés ou non, émanant des établissements d'enseignement et de recherche français ou étrangers, des laboratoires publics ou privés. 


\section{AULU-GELLE, PROBUS ET LE PROBLÈME DE LA TRADUCTION DES TEXTES POÉTIQUES}

Alessandro Garcea

in Bernard Bortolussi et al., Traduire, transposer, transmettre dans l'Antiquité grécoromaine

Editions Picard | «Textes, images et monuments de l'Antiquité au Haut Moyen

Âge »

2009 | pages 17 à 26

ISBN 9782708408388

Article disponible en ligne à l'adresse :

https://www.cairn.info/traduire-transposer-

transmettre--9782708408388-page-17.htm

Distribution électronique Cairn.info pour Editions Picard.

(C) Editions Picard. Tous droits réservés pour tous pays.

La reproduction ou représentation de cet article, notamment par photocopie, n'est autorisée que dans les limites des conditions générales d'utilisation du site ou, le cas échéant, des conditions générales de la licence souscrite par votre établissement. Toute autre reproduction ou représentation, en tout ou partie, sous quelque forme et de quelque manière que ce soit, est interdite sauf accord préalable et écrit de l'éditeur, en dehors des cas prévus par la législation en vigueur en France. Il est précisé que son stockage dans une base de données est également interdit. 


\title{
AULU-GELLE, PROBUS ET LE PROBLÈME DE LA TRADUCTION DES TEXTES POÉTIQUES
}

\author{
par ALESSANDRO GARCEA*
}

\section{Remarques théoriques préliminaires}

Le caractère hétérogène et souvent indéterminé des critères réglant les méthodes de traduction et de transposition dans l'Antiquité gréco-latine a bien été mis en évidence, tout dernièrement, par la dissertation d'Astrid Seele (1995), qui se fonde sur la distinction sémiotique entre les phénomènes sémantico-lexicaux, les questions syntaxiques et stylistiques, et les aspects pragmatiques qui relèvent du rapport entre l'auteur et le destinataire, ainsi que de la réception culturelle d'une œuvre. Si la pertinence heuristique d'une telle démarche ne saurait être sous-estimée, il nous paraît opportun de mettre en valeur le rôle complémentaire de la répartition en genres littéraires, et les conclusions différentes auxquelles l'on peut parvenir en fonction du type de texte que l'on choisit comme objet d'étude. Plus particulièrement, dans cet article, nous souhaitons attirer l'attention sur quelques problèmes posés par la définition des principes de traduction des textes poétiques, dont la composante «esthétique» s'avère non seulement peu définissable, mais aussi, et à plus forte raison, peu transposable d'une langue à une autre par un processus mécanique ${ }^{1}$. En effet, la poésie oblige son contenu à se conformer à une structure bien précise: elle recrée le contenu lui-même en fonction des exigences de l'expression, suivant une norme que, à l'opposé de celle qui sous-tend l'écriture en prose, on pourrait formuler comme: uerba tene, res sequentur ${ }^{2}$. Dans le cadre de la traduction d'un texte poétique, pourvu qu'elle soit possible, il faut par conséquent trouver un moyen de respecter les impératifs de la forme que sont les effets de son, les figurae elocutionis, les schémas métriques, etc., dans la mesure où ils renvoient au contenu, suivant un mouvement systématique d'aller-retour entre les deux niveaux.

\footnotetext{
* Université de Toulouse 2, UMR 7597 (Université de Paris 7), agarc@libero.it

1. Cf. notamment Eco (2003/2007: § 11.4-5).

2. Ainsi Eco (1985).
} 


\section{Aulu-Gelle 9,9,1-3: les critères de la traduction d'un texte poétique}

L'intérêt particulier qu'Aulu-Gelle porte aux problèmes de traduction a bien été étudié par Leopoldo Gamberale dans une monographie qui fait toujours autorité et dont les résultats peuvent être résumés comme suit: «Nel passo, o nell'opera greca, vi sono certe caratteristiche: la traduzione deve cercare di rispettarle e di riprodurle, e pertanto i giudizi che si possono dare su di essa vertono [...] soprattutto sulle omissioni, sui tagli, sugli ampliamenti, sulle corruzioni che l'opera del traduttore ha fatto subire all'originale» (1969: 63). Par conséquent, «l'esercizio è compiuto prevalentemente secondo norme definite: la riproduzione quanto più è possibile fedele delle caratteristiche del greco, e per alcuni particolari la ricerca di una corrispondenza ad uerbum [...] Quanto più il latino si distacca dall'originale, tanto più perde valore, in quanto è sempre considerato un'imitazione, una versione soprattutto» (ibid.: 169-171). Une démarche critique similaire, même si le point de vue de son auteur n'est pas exactement le même, est encore présupposée par l'important article d'Amiel D.

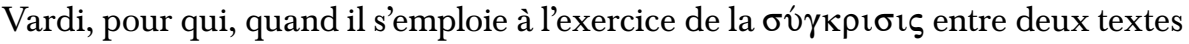
d'un auteur grec et d'un auteur latin, Aulu-Gelle semble concevoir l'imitatio du premier par le second selon une perspective figée, ex negatiuo: «This evaluative technique [...] involves judgement of only one of the passages, the imitation, and can therefore never find it superior to his model. Furthermore, [...] it is always the imitation (or "translation") which is required to correspond to the original and not the other way round. And finally, the quality of similitudes between the passages [...] is altogether irrelevant to syncritic evaluation, in which the respective value of the passages is judged by external criteria» (1996: 504).

Et pourtant, sans oublier les résultats partiels auxquels était déjà parvenu Peter Steinmetz (1992), Stephen M. Beall (1997) a clairement montré que les questions de rythme et de style occupent une place tellement essentielle dans les traductions d'Aulu-Gelle lui-même que cet auteur est souvent disposé à s'éloigner de la physionomie lexicale et syntaxique de son modèle pour produire des versions latines artistiquement soignées ${ }^{3}$. Par exemple, dans le cas de la lettre de Philippe de Macédoine à Aristote, citée en 9,3,5-6, "While retaining the formal structure of his model, he altered its syntax and modulated its vocabulary in the interest of smoothness and stylistic equivalence» (ibid.: 221). Ailleurs, comme dans l'histoire d'Arion et du dauphin (16,19), Aulu-Gelle va jusqu'à concevoir un style personnel, lui permettant de se démarquer de ses modèles, Hérodote et Fronton. Cette autonomie semble encouragée, entre autres, par l'apprentissage des principes rhétoriques de la traduction artistique, tels qu'ils avaient été exposés par exemple dans le De optimo genere oratorum de Cicéron:

c o n u erti enim ex Atticis duorum eloquentissimorum nobilissimas orationes inter seque contrarias, Aeschinis et Demosthenis; nec c o n u e r t $i$ ut interpres ${ }^{4}$, sed ut orator,

3. Cf. également l'excellente mise au point de Holford-STrevens (2007).

4. Cf. YON (1964: 103 n. 1): «Interpres désigne d'abord en latin l'intermédiaire et le courtier qui 
sententiis isdem et earum formis tamquam figuris ${ }^{5}$, uerbis ad nostram consuetudinem aptis. in quibus non uerbum pro uerbo necesse habui reddere, sed genus omne uerborum ${ }^{6}$ uimque seruaui. non enim ea me annumerare lectori putaui oportere, sed tamquam appendere. (Cicéron opt. gen. 14)

Certes, dans ce domaine, les préceptes ne peuvent qu'être plus indéterminés et plus subjectifs que ceux qui valent pour la traduction littérale, car les compétences et la sensibilité du traducteur y jouent un rôle déterminant, mais ce qui s'avère essentiel, c'est le respect d'une double fidélité, au grec sur le plan conceptuel des sententiae et de l'enchaînement des arguments, au système linguistique latin sur le plan formel, celui des uirtutes expressives qui font la valeur esthétique d'un texte. Ces deux aspects sont à nouveau évoqués à la fin du petit traité $^{7}$ :

quorum ego orationes si, ut spero, ita exp re s s e ro uirtutibus utens illorum omnibus, $i d$ est sententiis et earum figuris et rerum ordine, uerba persequens eatenus ut ea non abhorreant a more nostro (quae si e Graecis omnia c o n u e r s a non erunt, tamen ut generis eiusdem sint, elaborauimus), erit regula, ad quam eorum dirigantur orationes, qui Attice uolent dicere. (Cicéron opt. gen. 23)

Dans l'étude théorique de la traduction artistique la poésie devait revêtir la plus grande importance. Cicéron lui-même se préoccupe à plusieurs reprises de souligner la contiguïté entre celle-ci et l'éloquence, l'exercice de traduire des poètes grecs permettant d'explorer toutes les ressources de la langue latine et d'en sonder les potentialités au niveau stylistique le plus élevé ${ }^{8}$. C'est ainsi que l'on voit réapparaître la même doctrine dans une œuvre d'érudition comme les Noctes Atticae, dont l'intérêt pour les questions linguistiques s'avère prioritaire. Dans le lemma de 9,9 ainsi qu'en ouverture du même chapitre, Aulu-Gelle affirme:

quis modus sit u e r te n $i$ uerba in Graecis sententiis; deque his Homeri uersibus, quos Vergilius u e $t$ t $s$ se aut bene apteque aut inprospere existimatus est. 1. quando ex poematis Graecis u e r te n d a e $i$ m it a n d a e q u e sunt insignes sententiae, non semper aiunt enitendum, ut omnia omnino uerba in eum, in quem dicta sunt, modum u e $r$ t a $m$ u $s$. 2. perdunt enim gratiam pleraque, si quasi inuita et recusantia uiolentius $t \mathrm{r}$ a $n \mathrm{~s} f \mathrm{e}$ r a $n$ t $u$ r. 3. scite ergo et considerate Vergilius, cum aut Homeri aut Hesiodi aut Apollonii aut Parthenii aut Callimachi aut Theocriti aut quorundam aliorum locos effingeret, partem reliquit, alia exp ress it. (Aulu-Gelle 9,9,1-3)

s'entremet entre les parties dans le domaine des affaires [...]. Son emploi ici en opposition avec orator précise le rôle du traducteur: il n'est qu'un interprète s'il se borne à donner objectivement le sens du texte».

5. Cf. Yon (1964: 163 n. 2 de p. 114 ligne 16): «C'est le groupement formis tamquam figuris qui désigne ici les figures [sc. de pensée, A.G.], le premier mot n'ayant pas une valeur technique suffisamment précise et le second n'étant pas encore reçu dans ce sens».

6. Cf. Yon (1964: 163 n. 3 de p. 114 ligne 19): «Dans leur sens plus large, les figures de mots [...]. Celles-ci sont intraduisibles d'une langue à l'autre; mais le traducteur soucieux de rendre non seulement le sens des mots, mais aussi leur valeur stylistique, doit s'efforcer d'en donner l'équivalent».

7. Cf. Yon (1964: 102-105).

8. Cf. par ex. de orat. 1,70; Leeman \& Pinkster (1981 ad loc.). Sur l'importance de la poésie dans la formation d'un orateur, cf. notamment North (1952); sur la théorie de la traduction chez Cicéron: Serra Zanetti (1961: 357-367); Traina (1961/1974²: 57-65). 
Amiel D. Vardi (1996: 505) observe à juste titre que le mot aiunt du $\S 1$ fait allusion à une doctrine courante qui devait être transmise par les rhéteurs. En effet, malgré les différences entre la perspective rhétorico-philosophique de Cicéron et une œuvre de compilation comme les Noctes Atticae, il est évident que la position d'Aulu-Gelle en 9,9,1-3 ne s'éloigne pas beaucoup de la démarche théorique de l'Arpinate ${ }^{9}$.

L'exemple le plus représentatif pour les élèves étant probablement celui de la traduction artistique réalisée par Virgile, Aulu-Gelle saisit l'occasion de la lecture parallèle de Théocrite et des Bucoliques pendant un banquet pour suggérer les deux $\sigma \mho \gamma \kappa \rho i ́ \sigma \varepsilon ı \varsigma$ suivantes ${ }^{10}$ :

1. Théocrite 5,88-89 et Virgile ecl. 3,64-65:

animaduertimus reliquisse Vergilium, quod Graecum quidem mire quam suaue est, uerti autem neque debuit neque potuit. 5. sed enim, quod substituit pro eo, quod omiserat, non abest, quin iucundius lepidiusque sit.

2. Théocrite 3,3-5 et Virgile ecl. 9,23-25:

7. illud quoque alio in loco animaduertimus caute omissum, quod est in Graeco uersu dulcissimum. [...] 9. hoc igitur reliquit et cetera uertit non infestiuiter, nisi quod "caprum" dixit, quem Theocritus ع́vó $\chi \alpha \varsigma$ appellauit 10. - auctore enim M. Varrone is demum Latine caper dicitur, qui excastratus est.

Dans ces deux comparaisons, Aulu-Gelle s'exprime à la première personne et, notamment dans le cas de la troisième Bucolique, il émet un jugement entièrement positif à l'égard du poète latin, car les omissions et les substitutions qu'il a opérées comportent un résultat plus agréable (iucundius lepidiusque) que

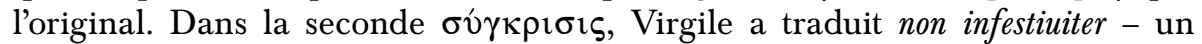
hapax d'Aulu-Gelle ${ }^{11}$ - les vers des Théocrite, en se passant de la locution $\tau$ ò $\kappa \alpha \lambda$ òv $\pi \varepsilon \varphi \imath \lambda \eta \mu \varepsilon ́ v \varepsilon$, qui - si elle est dotée d'une natiua dulcedo (§ 8) - ne peut être transposée en latin. La seule remarque négative porte sur l'équivalence entre les mots દ̇vó $\chi \chi \alpha \varsigma$ et caper, ce dernier désignant le seul animal châtré.

9. Cf. Reiff (1959: 100-101); contra: Gamberale (1969: 105 n. 85) qui, d'abord, remarque la différence entre les genres littéraires dont relèvent les œuvres cicéroniennes, d'une part, et les traductions poétiques évoquées par Aulu-Gelle, d'autre part; ensuite, propose un rapprochement avec Horace ars 133-134, passage qui toutefois, comme le remarque MARAche (1978: 216 n. 1 à la p. 126), «ne s'occupe pas seulement de traduction, mais de création poétique à partir d'un modèle grec». Les correspondances conceptuelles entre Cicéron et Aulu-Gelle sont corroborées par le recours à un lexique technique partiellement identique, plus riche chez Aulu-Gelle. La traduction mot à mot est désignée par le verbe $e x p$ r $i$ m e re, employé neuf fois dans les Noctes, dont quatre dans le sens de «traduire à la lettre» (au-delà de 9,9 , cf. 1,10,9;4,5,7;11,16,3), probablement à la suite d'un usage déjà bien affirmé (cf. Richter 1938: 14; Gamberale 1969: 96 et n. 70); u e r t e r e , qui dès le lemma du chapitre, désigne génériquement chez Aulu-Gelle l'œuvre de traduction, aussi bien de sententiae (cf. lemma, § 1 et 12) que de mots (cf. lemma, § 2 et 9), alors que les occurrences assez limitées de ce verbe dans les textes précédents assument des valeurs plus spécifiques (cf. Richter 1938: 11-12; GAMBERALE 1969: 106 n. 88), et que Cicéron préfère au verbe simple le composé c o n u e r te re ; effing e re désigne la reproduction des caractéristiques formelles de l'original (cf. 8,8 lemma; 17,20,8), alors que dans le De oratore cicéronien le même verbe s'applique à l'imitation d'auteurs de la même langue $(2,90$; cf. GAMBERALE 1969: 99-100 n. 74); $t$ r a $n$ s f e r $r$ e, un verbe qui n'a peut-être pas de valeur particulière, et qui s'avère un simple synonyme de uertere (cf. 9,9,2 et 12; 17,10,15; RICHTER 1938: 10-11; GAMBERALE 1969: 112 n. 100).

10. Sur ces deux passages cf. Gamberale (1969: 108-111).

11. Cf. Marache (1957: 219); Gamberale (1969: 109 n. 94). 
Et pourtant, malgré un témoignage de Varron (frg. 419 Funaioli = 104 Goetz \& Schoell) allant dans le même sens, ce vers ne constitue pas le seul exemple virgilien - ni latin tout court - où caper est employé comme synonyme de hircus, pour indiquer le mâle du troupeau ${ }^{12}$.

Leopoldo Gamberale remarque «il carattere del tutto negativo di questi giudizi, che sono impostati e si soffermano soltanto sulle divergenze, evitando di dare rilievo a particolari cure, anche evidenti, del traduttore, nella resa stilistica del modello» (1969: 111). De même, Amiel D. Vardi estime que les critères présentés par Aulu-Gelle «Do not regard divergence from the original as a requirement which furnishes the imitator with the opportunity to exhibit his own achievement, but rather as a concession to the difficulty (and at times impossibility) of full correspondence to the original» (1996: 505-506). Ces jugements, sans être faux, ne nous paraissent pas tenir compte du préambule du chapitre 9,9, qui s'ouvre précisément par une exhortation à s'éloigner du modèle, démarche qui est par la suite vérifiée dans les textes, et qui se retrouve d'ailleurs dans la pratique de la traduction suivie par Aulu-Gelle lui-même.

\section{L'extrait de Probus et la méthode du grammaticus}

Le caractère excessivement sévère des critiques émises par les commentateurs à l'égard d'Aulu-Gelle semble dériver, d'une part, du silence de celui-ci sur ses propres méthodes de travail, d'autre part, d'une rétroprojection de l'avis des disciples de Probus, contenu dans la seconde partie de 9,9, sur l'auteur de ce chapitre.

Les § 12-17 contiennent une série de remarques portant sur les vers de l'Énéide (1,498-502) où Virgile adapte la comparaison homérique entre Nausicaa et Diane $\left(O d\right.$. 6,102-108) à la reine Didon ${ }^{13}$. Même si le nom de Probus figure seulement au $\S 12$, les exégètes ont été enclins à reconnaître dans ce grammairien la source unique du chapitre: ainsi, Aistermann publie les § 1-11 comme frg. $\mathrm{V}^{14}$ de son édition et la section des $\S 12-17$ comme frg. 4 . Sans revenir une nouvelle fois sur cette question ${ }^{15}$, nous nous limiterons à une étude des critères qui fondent un jugement esthétique selon l'école de Probus.

Touchant au problème des omissions $(\S 15-16)$ et des correspondances erronées $(\S 15)$, les réserves de Probus semblent émaner - comme l'a partiellement suggéré Aistermann (1910: 13. 62-63) - d'exercices préparatoires comme celui

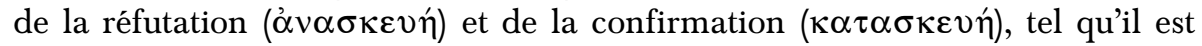

12. Cf. par ex. Virgile ecl. 7,7. 9; Horace epod. 10,23; Holford-Strevens (20032: 204 n. 42).

13. Sur l'histoire de ce passage dans la doxographie ancienne et moderne, cf. notamment GLEI (1990); pour Aulu-Gelle: Gamberale (1969: 112-116); Holford-Strevens (2003²: 201-203).

14. Le chiffre romain caractérise «ea quae Probi nomine destituta ad hunc grammaticum referenda esse uidebantur».

15. Comme le remarque justement Holford-Strevens $\left(2003^{2}: 74-75\right)$, «The critic who makes Probus the source for the whole chapter should specify whether the favourable judgments were his too, or only the parallels themselves. The pedantic Probus of $§ \S 12-17$ [...] seems less likely to perceive new beauties than to miss old ones». Pour cette raison, «we should take the passage at face value as a postcript, one of many in the Nights, appended "while we are on the subject" ("et quoniam de transferendis sententiis loquor") attached to matter independent of it». 
illustré par exemple par Aelius Théon ${ }^{16}$. Dans cette forme de progymnasmata,

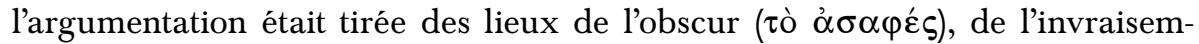

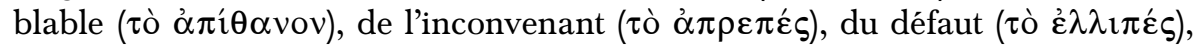

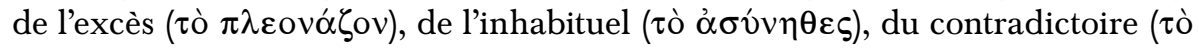

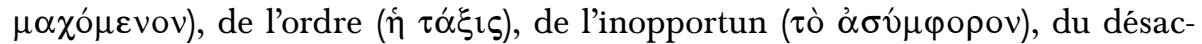

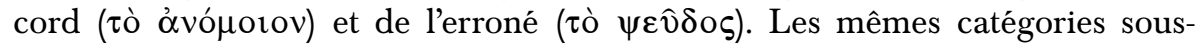
tendent les remarques suivantes:

1. si la comparaison entre Nausicaa et Diane est pertinente, celle entre Didon et la déesse ne respecte pas le $\pi \rho \varepsilon \pi_{0} v^{17}$ :

14. primum omnium id uisum esse dicebant Probo, quod aput Homerum quidem uirgo Nausica ludibunda inter familiares puellas in locis solis recte at que co m mode confertur cum Diana uenante in iugis montium inter agrestes deas, nequaquam autem c o n u e nie ns Vergilium fecisse, quoniam Dido in urbe media ingrediens inter Tyrios principes cultu atque incessu serio,

«instans operi - sicut ipse ait - regnisque futuris» [Aen. 1,504],

nihil eius similitudinis capere possit, quae lusibus atque uenatibus Dianae congruat.

2. Virgile ne faisant pas de référence à la chasse, la mention du carquois n'a pas de raison de subsister et, telle qu'elle figure dans le passage en question, s'oppose à la $\sigma \alpha \varphi \eta ́ v \varepsilon ı \alpha^{18}$ :

15a. tum postea, quod Homerus studia atque oblectamenta in uenando Dianae $h$ o $n$ e s te a p e r te que dicit, Vergilius autem, cum de uenatu deae nihil dixisset, pharetram tantum facit eam ferre in humero, tamquam sit onus et sarcinam.

3. la manière dont Virgile indique la joie de Leto constitue un exemple de $\tau \alpha \pi \varepsilon i ́ v \omega \sigma \imath \varsigma$, le défaut de l'expression qui ravale la grandeur d'un sujet, car pertemptare est un verbe sans éclat ${ }^{19}$ :

15b. atque illud impense Probum esse demiratum in Vergilio dicebant, quod Homerica quidem $\Lambda \eta \tau \omega ́$ gaudium gaudeat genuinum et intimum atque in ipso penetrali cordis et animae uigens, siquidem non aliud est:

$\gamma \dot{\varepsilon} \gamma \eta \theta \varepsilon \delta \dot{\varepsilon} \tau \varepsilon \phi \rho \varepsilon \dot{v} \alpha \Lambda \eta \tau \omega ́$ [Od. 6,106],

ipse autem imitari hoc uolens gaudia fecerit $p i g$ ra et le u $i$ a et cunct antia et quasi in summo pectore s u p e r n a n t i a ; 16. nescire enim sese, quid significaret aliud "pertemptant".

4. enfin, Virgile néglige la tournure essentielle du texte-source; cette omission, au lieu d'être interprétée en fonction du principe général qui veut que la poésie n'est pas entièrement traduisible $(\S 1)$, reçoit les plus âpres

16. Cf. Aelius Théon p. 76,7-78,14 Spengel = 35-38 Patillon et cf. PAtillon (1997: XCIII-XCVII). La section que Quintilien consacre à l'opus destruendi confirmandique (inst. 2,4,18-19) est beaucoup plus succincte.

17. Cf. Aelius Théon prog. p. 76,6-12 et 76,34-77-10 Spengel $=35$ et 36-37 Patillon.

18. Cf. Aelius Théon prog. p. $76,25-34$ et $81,4-7$ Spengel $=36$ et 42 Patillon.

19. Cf. Aelius Théon prog. p. $78,9-13$ Spengel $=38$ Patillon. Sur la $\tau \alpha \pi \varepsilon i ́ v \omega \sigma \imath \varsigma$, cf. par ex. Quintilien inst. 8,3,48-49. 
critiques, car le poète montrerait ainsi ne pas avoir saisi la fonction centrale de la comparaison:

praeter ista omnia florem ipsius totius loci Vergilium uideri omisisse, quod hunc Homeri uersum exigue secutus sit:

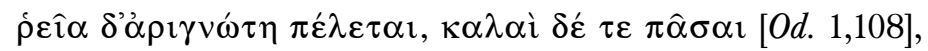

17. quando nulla maior cumulatiorque pulcritudinis laus dici potuerit, quam quod una inter omnis pulcras excelleret, una facile ex omnibus nosceretur.

Que ce soit dans le cadre d'une édition critique ou non, et en dépit du silence de Suétone, au chapitre 24 de son De grammaticis, Probus s'est certainement appliqué à annoter le texte de Virgile: un passage corrompu de l'anecdoton Parisinum (GL 7,534,4-6) semble faire allusion à l'insertion de signes diacritiques dans les poèmes de Virgile, d'Horace et de Lucrèce par ce grammairien. Certaines de ces adnotationes, issues des préceptes du rhéteur Théon d'Alexandrie, correspondent aux catégories d'évaluation que nous venons d'évoquer (GL $7,536,17-23)^{20}$ :

\begin{tabular}{|c|c|}
\hline NOTAE SIMPLICES & тоПоI CHEz AELIUS ThÉON \\
\hline M malum metrum aut aprepes & 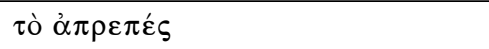 \\
\hline П contra historiam & $\tau o ̀ ~ \alpha ̉ \pi i ́ \theta \alpha \nu o v$ \\
\hline$\Theta$ superuacuus & 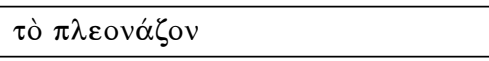 \\
\hline - repugnans & $\tau o ̀ ~ \mu \alpha \chi o ́ \mu \varepsilon v o v$ \\
\hline — praepositum sine consequente & \\
\hline - consequens sine praeposito & $\dot{\eta} \tau \alpha \xi_{\imath \jmath \varsigma}$ \\
\hline
\end{tabular}

Mais ce sont surtout le commentaire de Servius à Virgile et les Scholia Danielis qui, citant trente-neuf fois Probus pour ses remarques sur la langue et sur le style du poète, pour les leçons qu'il a proposées, pour ses explications et pour ses jugements esthétiques (cf. test. 37 et frg. 3-42 Aistermann), semblent corroborer l'idée qu'il y eut au moins un manuscrit de Virgile annoté par Probus ainsi que, probablement, des dissertations où ce grammairien justifiait ses choix, œuvres auxquelles Donat et Servius auraient puisé pour rédiger les leurs ${ }^{21}$.

\section{Aulu-Gelle 2,6}

Une confirmation de la méthode utilisée par les grammairiens pour commenter les textes sur les plans linguistique, grammatical et rhétorique vient du chapitre 2,6 des Noctes, où Aulu-Gelle examine les critiques émises par certains detractores Vergili, notamment par Annaeus Cornutus dans ses Commentaria in Vergilium (frg. 22 Mazzarino $\left.^{2}\right)^{22}$, pour ensuite les réfuter, suivant un schéma figé:

20. Cf. Aistermann (1910: 11-12).

21. La question est extrêmement controversée: on peut se reporter à Timpanaro (2001: 37-105) pour un remarquable essai de synthèse.

22. Cf. aussi Aulu-Gelle 9,10 pour une critique de l'avis que Cornutus avait émis sur Aen. 8,404-406 
citation des passages du poète, compte rendu des critiques ponctuelles, réplique tout autant exacte d'Aulu-Gelle. Parmi les vers examinés, il convient de s'arrêter sur ecl. 6,75-77, la description du monstre marin Scylla qui s'emparait des navires pour les noyer:

VEXASSE enim putant uerbum esse le u e e $t$ t e $n$ u $i$ s ac parui incommodi nec tantae atrocitati congruere, cum homines repente a belua immanissima rapti laniatique sint. (AuluGelle 2,6,2).

De même que pertemptare de 9,9,15, l'expression uexasse rates est jugée comme inadéquate et trop faible pour décrire l'action de Scylla. La même considération se trouve dans le commentaire de Servius, qui estime que uexare est un verbe sans éclat et pour ainsi dire minimisant:

VEXASSE RATES autem per t a p i n o s $i$ n dictum est; nam non uexauit sed euertit (3 p. 80,6-7 Thilo \& Hagen).

Mais dans la deuxième partie de sa glose, Servius (p. 80,7-13 Thilo \& Hagen), comme Aulu-Gelle en 2,6,5-6, justifie l'usage virgilien de uexare à partir de l'interprétation qu'en donne Probus (frg. 3 Aistermann), pour qui uexare vient de uehere ${ }^{23}$ :

\begin{tabular}{|c|c|}
\hline Aulu-Gelle & SERVIUS + SCHOLIA DANIELIS \\
\hline $\begin{array}{l}\text { a) sed de uerbo "uexasse" ita responderi posse } \\
\text { credo: "uexasse" graue uerbum est factumque } \\
\text { ab eo uidetur, quod est "uehere", } \\
\text { b) in quo inest uis iam quaedam alieni arbi- } \\
\text { trii; non enim sui potens est, qui uehitur. } \\
\text { c) "uexare" autem, quod ex eo inclinatum est, } \\
\text { ui atque motu procul dubio uastiorest. } \\
\text { d) nam qui fertur et rapsatur atque huc atque } \\
\text { illuc distrahitur, is uexari proprie dicitur. }\end{array}$ & $\begin{array}{l}\text { a) quod Probus uult hac ratione defendere, } \\
\text { dicens "uexasse" uenire ab eo, quod est "ueho, } \\
\text { uecto, uexo", ut "uexasse" sit portasse et sine } \\
\text { dubio pro arbitrio suo euertisse. } \\
\text { b) "uexasse" est enim uis quaedam alienii } \\
\text { arbitrii, non enim sui potens est, qui uehitur. } \\
\text { c) bene ergo inclinatum uerbum est; } \\
\text { d) nam qui fertur et raptatur et huc atque illuc } \\
\text { distrahitur, "uexari” proprie dicitur. }\end{array}$ \\
\hline
\end{tabular}

S'il ne mentionne pas explicitement le nom de Probus, l'identité entre son texte et celui du scholiaste amène à croire qu'Aulu-Gelle avait utilisé des matériaux remontant au grammairien de Beyrouth. Cette hypothèse est d'autant plus vraisemblable que la partie suivante des extraits du tableau comprend des exemples tirés de Caton (De Achaeis frg. 142 Sblendorio Cugusi) et de Cicéron (Verr. 2.4,104 et 122), dont la version des scholia Danielis, qui mentionnent explicitement Probus, s'avère plus complète, intégrée de surcroît par un second passage des Verrines ( $\$ 104$ ) absent des Noctes. Aulu-Gelle ne peut donc être tenu pour la source des scholies qui, comme lui, reflètent la doctrine d'une source commune: Probus.

dans son De figuris sententiarum (frg. 36 Mazzarino $^{2}$ ).

23. Cf. aussi Macrobe Sat. 6,7,4-11; REPPE (1906: 35-36). 


\section{Aulu-Gelle et Probus}

La comparaison entre la première et la seconde section de 9,9 permet de conclure que, même s'il se montre au courant de la théorie rhétorique sur la traduction artistique, Aulu-Gelle considère celle-ci uniquement dans les paragraphes initiaux du chapitre. Dans le reste, comme dans d'autres $\operatorname{cas}^{24}$, il estime que la fidélité à l'original constitue la qualité essentielle d'une bonne traduction. Le jugement de René Marache (1952: 307), pour qui les remarques de l'érudit latin «ne cherchent pas à revêtir une portée générale, elles restent accrochées au mot qui en a été le prétexte et asservies au détail», même s'il ne s'avère pas complètement erroné, est du moins assez restrictif: puisque les critères formels l'emportent sur tout le reste, et malgré le fait qu'à partir du $\S 12$ ce n'est plus lui qui évalue la traduction de Virgile, Aulu-Gelle semble quand même suivre

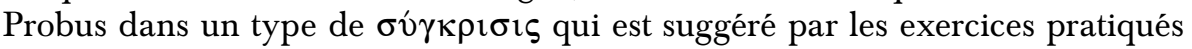
dans les écoles.

Cela étant, si aussi bien en 2,6 qu'en 9,9 il préfère appliquer la méthode scolaire de discussion des textes pour mettre en valeur ses compétences linguistiques, en se reportant dans les deux cas à l'avis de Probus, en 2,6 il prend position pour défendre Virgile, en 9,9 il ne dit rien après la citation des remarques négatives du grammairien. Ce silence est d'autant plus étonnant que Virgile constitue un auteur très apprécié par Aulu-Gelle, qui le loue presque toujours dans ses Noctes $^{25}$. Vraisemblablement, s'abstenant de toute considération personnelle, celui-ci devait indiquer de manière implicite son adhésion - certes non enthousiaste - aux réserves de Probus, sans pour autant prendre position contre le poète qu'il aimait. Entre le point de vue d'Aulu-Gelle et celui du grammairien de Beyrouth il y a donc des nuances, dont il faut nécessairement tenir compte dans la reconstitution de la mouvance archaïsante, où rentrent des personnalités distinctes qu'il serait inapproprié de confondre à cause de leur intérêt commun pour les plus anciens représentants de la littérature latine.

24. Cf. Aulu-Gelle 13,27, une comparaison entre, d'une part, Parthenius (frg. 20 Diehl = 647 Lloyd Jones \& Parsons) et Homère $I l$. 11,728, et, d'autre part, Virgile georg. 1,437 et Aen. 3,119.

25. Cf. Gamberale (1985). 


\section{Bibliographie}

Aistermann Josef, 1910, De M. Valerio Probo Berytio capita quattuor, Bonnae, In aedibus Cohen.

Beall Stephen, 1997, «Translation in Aulus Gellius», Classical Quarterly, 47, 215-226.

Eco Umberto, 1985, «Il segno della poesia e il segno della prosa", Id., Sugli specchi e altri saggi, Milano, Bompiani, 242-260.

Eco Umberto, 2003/2007, Dire quasi la stessa cosa. Esperienze di traduzione, Milano, Bompiani, tr. fr. Paris, Grasset.

Gamberale Leopoldo, 1969, La traduzione in Gellio, Roma, Edizioni dell'Ateneo.

Gamberale Leopoldo, 1985, «Gellio, Aulo", Enciclopedia virgiliana, 2, Roma, Istituto dell'Enciclopedia Italiana, 643-645.

GleI Reinhold, 1990, «Von Probus zu Pöschl: Vergilinterpretation im Wandel», Gymnasium, 97, 321-340.

HolfOrd-STRevens Leofranc, $2003^{2}$, Aulus Gellius. An Antonine Scholar and his Achievement, Oxford, University Press.

Holford-Strevens Leofranc, 2007, «An Antonine Littérateur: The Case of Aulus Gellius», H. Kittel et alii (éd.), Übersetzung-Translation-Traduction. Ein internationales Handbuch zur Übersetzungsforschung, Berlin/New York, de Gruyter, 1143-1150.

Lehmann Anton D. \& Pinkster Harm, 1981, M. Tullius Cicero. De oratore libri III, 1, Heidelberg, Winter.

MARAche René, 1952, La critique littéraire de langue latine et le développement $d u$ goût archaïsant au II siècle de notre ère, Rennes, Plihon.

Marache René, 1957, Mots nouveaux et mots archaïques chez Fronton et AuluGelle, Paris, Presses universitaires de France.
Marache René, 1978, Aulu-Gelle. Les nuits attiques, II, Liores V-X, Paris, Les Belles Lettres.

North Helen, 1952, «The Use of Poetry in the Training of the Ancient Orator», Traditio, 8, 1-33.

Patillon Michel, 1997, Aelius Théon. Progymnasmata, Paris, Les Belles Lettres.

ReIFF Arno, 1959, Interpretatio, imitatio, aemulatio. Begriff und Vorstellung literarischer Abhängigkeit bei den Römern, Diss. Köln.

Repre Rudolf, 1906, De L. Annaeo Cornuto, Diss. Leipzig.

Richter Hans Eberhard, 1938, Uebersetzen und Uebersetzungen in der römischen Literatur, Diss. Erlangen.

SEelE Astrid, 1995, Römische Übersetzer, Nöte, Freiheiten, Absichten: Verfahren des literarischen Übersetzens in der griechisch-römischen Antike, Darmstadt, Wissenschaftliche Buchgesellschaft.

Serra Zanetti Paolo, 1961, «Sul criterio e il valore della traduzione per Cicerone e san Girolamo", Atti del I congresso di studi ciceroniani, 2, Roma, Centro di Studi Ciceroniani, 355-405.

Steinmetz Peter, 1992, "Gellius als Übersetzer», C.W. Muller, K. Sier \& J. Werner (éd.), Zum Umgang mit fremden Sprachen in der griechisch-römischen Antike, Stuttgart, Steiner, 201-211.

Timpanaro Sebastiano, 2001, Virgilianisti antichi e tradizione indiretta, Firenze, Olschki.

Traina Alfonso, 1961/19742, «Commento alle traduzioni poetiche di Cicerone», Atti del I congresso di studi ciceroniani, 2, Roma, Centro di Studi Ciceroniani, 141-159 = Id., Vortit barbare, Roma, Edizioni dell'Ateneo, 55-89.

VARDI Amiel, 1996, «Diiudicatio Locorum: Gellius and the History of a Mode in Ancient Criticism », Classical Quarterly, 46, 492-514. 